\title{
Optic disc swelling in a patient with tuberculous meningitis: a diagnostic challenge
}

\author{
Hanis Zuhaimy, Sue Ngein Leow, Suresh K Vasudevan
}

Department of Ophthalmology, Hospital Sultanah Aminah, Johor Bahru, Johor, Malaysia

\section{Correspondence to} Dr Hanis Zuhaimy, hanis_zuhaimy@hotmail.com

Accepted 30 July 2017

To cite: Zuhaimy H, Leow SN, Vasudevan SK. BMJ Case Rep Published Online First: [please include Day Month Year]. doi:10.1136/bcr-2017221170

\section{SUMMARY}

We discuss the diagnostic challenge in an adult patient presented with purely ocular symptoms diagnosed with tuberculous meningitis (TBM). A 38-year-old woman presented with bilateral painless blurring of vision. There were bilateral sixth cranial nerve palsy and bilateral optic disc swelling. Optic nerve function tests were normal. Patient was lucid with no signs of meningism. Brain imaging were normal. She had a positive Mantoux test, high erythrocyte sedimentation rate but no clinical evidence of active pulmonary tuberculosis infection. Her Quantiferon-TB Gold in-tube test was negative. Cerebrospinal fluid analysis revealed a high opening pressure but no biochemical parameters to suggest TBM, hence she was treated as idiopathic intracranial hypertension. A diagnosis of TBM was finally made following a positive PCR for Mycobacterium tuberculosis. Her vision improved with reduction in optic disc swelling following antituberculous treatment.

\section{BACKGROUND}

Tuberculous meningitis (TBM) is a life-threatening form of central nervous system infection causing death and severe disability up to $50 \%-60 \%$ of affected patients. ${ }^{12}$ TBM has a subacute onset of symptoms with non-specific clinical signs that may persist for weeks, often making early diagnosis difficult. Ocular manifestation of TBM with vision impairment is often seen in paediatric population and late stages of adult TBM. ${ }^{2}$ We report an atypical presentation of TBM where the patient presented with only ocular symptoms alone with no typical signs of meningism and normal brain imaging. The case illustrates that papilloedema with blurring of vision can be the initial presenting symptoms of early stages of TBM. Early recognition and treatment improve outcome.

\section{CASE PRESENTATION}

A 38-year-old woman presented with subacute onset of painless blurring of vision in both eyes for 2 weeks duration. This was associated with diplopia which later resolved spontaneously. Otherwise, there were no other complaints such as fever, headache, stiff neck, vomiting, cough or any neurological symptoms. On examination, her best corrected visual acuity (BCVA) in both eyes was $6 / 36$. Her fundi examination revealed bilateral optic disc swelling with peripapillary shaped haemorrhages, tortuous vessels and dot-blot haemorrhages confined to the posterior pole of the left fundus (figure 1A). There were no other signs of uveitis such as vitritis, retinitis or choroiditis. Optic nerve function tests which included pupillary light reflexes, colour vision, visual fields and light brightness were normal in both eyes. There was no relative afferent pupillary defect. Otherwise, her vital parameters were normal. No signs of meningeal irritation in the form of neck rigidity or Kernig's sign was present. Higher mental functions were normal. Cranial nerve examination revealed initial left sixth cranial nerve palsy which subsequently involved the right side.

\section{INVESTIGATIONS}

Laboratory investigations showed a raised erythrocyte sedimentation rate (ESR) of $109 \mathrm{~mm}$ with Mantoux reading of $18 \mathrm{~mm}$ at 72 hours. Quantiferon-TB Gold in-tube test was negative. Other infectious and immunological workout including chest radiograph was also negative. Contrast-enhanced CT of the brain was normal with no evidence of intracranial mass, midline shift or meningeal enhancement. MRI of brain/orbit showed no dural and meningeal enhancement, no enhancing lesions in the brain and both optic nerves were normal. Magnetic resonance venography was negative for cerebral venous sinus thrombosis. Neurology team opinion was sought. A lumbar puncture was performed. Cerebrospinal fluid (CSF) analysis revealed a high opening pressure of $58 \mathrm{cmH}_{2} \mathrm{O}$ and a closing pressure of $39 \mathrm{cmH}_{2} 0$. The lumbar puncture was traumatic as CSF analysis revealed slightly blood-stained CSF. CSF was drained until the fluid became clear and colourless. CSF glucose was $3.8 \mathrm{mmol} / \mathrm{L}$ (normal range $2.5-5.6 \mathrm{mmol} / \mathrm{L}$ ) with a plasma glucose of $4.3 \mathrm{mmol} / \mathrm{L}$. CSF protein was $0.45 \mathrm{~g} / \mathrm{L}(0.15-0.45 \mathrm{~g} / \mathrm{L})$. Both CSF glucose and protein levels were within normal range suggesting normal biochemical parameters. CSF cytology revealed white blood cell (WBC) count of $8 \times 10^{9} /$ $\mathrm{L}$ (normal range $0-5 \times 10^{\wedge} 9 / \mathrm{L}$ and absence of lymphocytes. No malignant cells were detected. CSF gram stain showed no microorganism. CSF for acid fast bacilli (AFB) stain was negative. India ink stain and latex agglutination were also negative. CSF culture and sensitivity grew no fungal or bacterial organisms after 5 days of incubation.

\section{DIFFERENTIAL DIAGNOSIS}

A diagnosis of idiopathic intracranial hypertension (IIH) was made by the neurology team because the patient had papilloedema in the setting of an 


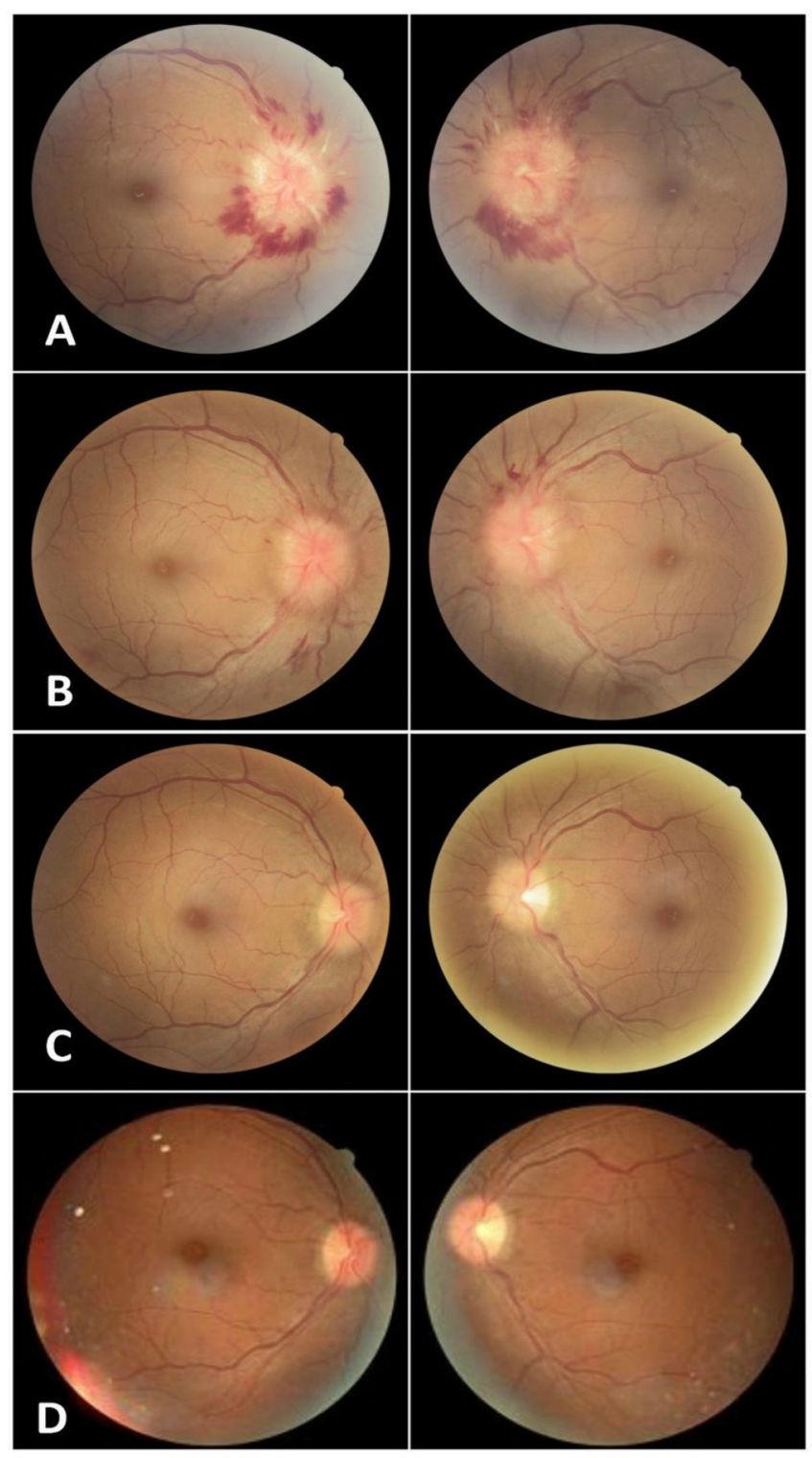

Figure 1 (A) Marked optic disc swelling with peripapillary haemorrhages, tortuous vessels in both fundi and dot blot haemorrhages confined to the posterior pole of left eye at presentation. (B) Reduction in both optic disc swelling and peripapillary haemorrhages after a month of antituberculosis treatment. (C) Gradual reduction of optic disc swelling in both fundi with resolution of peripapillary haemorrhages at 6 months of antituberculosis treatment. (D) Total resolution of optic disc swelling and peripapillary haemorrhages after 9 months of antituberculosis treatment with good visual acuity of 6/9 in both eyes.

increased intracranial hypertension; no other neurological deficits except for sixth cranial nerve palsy, normal brain imaging and a presumed normal CSF analysis, all of which fulfilled the criteria for the diagnosis of IIH. The slightly raised WBC count in CSF was attributed to the traumatic tap. However, they could not explain the high ESR in this case and they think it was unrelated and an incidental finding. Furthermore, clinically there were no obvious medical or laboratory findings to account for the high ESR. She had no clinical and laboratory evidence of malignancy, haematological disorders or autoimmune disease such as systemic lupus erythematosus and rheumatoid arthritis. She was started on oral acetazolamide $125 \mathrm{mg} 6$ hours daily and was discharged well after 2 weeks of hospitalisation pending results for CSF AFB culture and CSF PCR for tuberculosis.

\section{OUTCOME AND FOLLOW-UP}

Upon clinic review a week later, results of CSF PCR came back positive for Mycobacterium tuberculosis complex. A diagnosis of TBM was finally made. Antituberculosis treatment was commenced. She was given 2 months of isoniazid, rifampicin, pyrazinamide and ethambutol during the initiation phase. This was followed by 7 months of continuation phase consisting of isoniazid and rifampicin. CSF culture with the Lowenstein-Jensen media and BACTEC 460 came back negative 6-8 weeks later for M. tuberculosis. Her BCVA improved to 6/9 with marked reduction in optic disc swelling (figure 1C,D). There was no evidence of neurological sequelae, and optic nerve function in both eyes remained normal following completion of antituberculous treatment.

\section{DISCUSSION}

Diagnosis of TBM is based on clinical characteristics, laboratory, CSF findings and radiological imaging. However, the disease can prove to be a diagnostic challenge if there is no evidence of classical clinical findings. Diagnosis will be obvious once neurological signs of advanced disease are present but then the prognosis becomes poor. ${ }^{3}$

The most common presenting symptoms of TBM are fever, headache, vomiting and anorexia. Common clinical findings include neck rigidity, cranial nerve palsy, confusion, hemiparesis, seizures and coma. ${ }^{4}$ TBM is classified into three grades of severity according to the British Medical Research Council modified criteria. ${ }^{5}$ Stage I disease encompasses an alert and conscious patient with no neurological deficits (Glasgow coma scale (GCS)15). Stage II is manifestations of meningeal irritation with slight or no clouding of sensorium (GCS 14-11) and minor neurological deficit while stage III includes major neurological deficits with severe clouding of sensorium, convulsions and involuntary movements $(\mathrm{GCS}<10)$. This staging is used clinically to stratify patient and to predict prognosis. ${ }^{6}$

A review of 101 patients by Sinha et al reported that majority of patients (80\%) with TBM presented in late stages (stages II and III). ${ }^{2}$ Ocular signs such as cranial nerve palsy and papilloedema were seen in late stages of TBM, 52\% and 31\% respectively, ${ }^{2}$ with $27 \%$ of patients having visual impairment. Visual impairment of worse than $6 / 18$, papilloedema and cranial nerve palsy at initial presentation of TBM were found to be predictors of blindness and severe disability at 6 months. ${ }^{2}$ In our patient, the presentation of TBM was atypical. She presented in early stage of TBM with ocular signs of visual impairment, papilloedema and sixth cranial nerve palsy without classical signs of meningitis such as fever, headache, vomiting or stiff neck. Adverse outcomes such as blindness and disability at 6 months were not seen in our patient as she was started with antituberculosis treatment early.

In TBM, CSF analysis typically demonstrates lymphocyte predominant pleocytosis, elevated protein and low glucose. Sensitivity for single CSF analysis for AFB smear and culture is low unless repeat and large volumes of CSF are taken ${ }^{4}$ Common radiological findings such as meningeal enhancement, hydrocephalus, cerebral infarcts and tuberculomas also aid in the diagnosis of TBM. ${ }^{2-4}$

The fact that our patient had normal biochemical parameters in her CSF analysis and negative AFB smear made the diagnosis of TBM unlikely at the time together with a negative Quantiferon-TB Gold in-tube test. In addition, the negative neuroimaging 
findings further obscured the diagnosis. Increased intracranial pressure and papilloedema with normal neuroimaging point to a diagnosis of IIH. However, IIH with the presence of poor vision was unlikely in an acute setting. Visual acuity usually remains normal in patients with IIH with papilloedema except in longstanding papilloedema or when the central $10^{\circ}$ of the visual field is affected. ${ }^{7}$ Absence of relative afferent pupillary defect with normal visual fields, colour vision and light brightness in our patient indicated a normal optic nerve function, hence ruled out the diagnosis of tuberculous optic neuritis.

Sinha et al reported that out of the $27 \%$ of patients with TBM with vision impairment, optochiasmatic arachnoiditis and optochiasmal tuberculoma were the common causes detected with contrast MRI, $41 \%$ and $21 \%$ respectively. ${ }^{2}$ Other possible causes for vision impairment in TBM include optic nerve involvement by tuberculous lesion causing optic neuritis, tuberculous abscess, brain infarcts, hydrocephalus or ethambutol toxicity. ${ }^{2}$ In our patient, the neuroimaging findings were normal and showed no obvious tuberculous pathology to account for the patient's vision impairment. There was also no evidence of optic neuritis as optic nerve function test was normal. In our patient, the vision impairment could be caused by transient optic nerve head ischaemia and axoplasmic stasis. The transient optic nerve head ischaemia and axoplasmic stasis can happen either from direct compression or reduced perfusion of axons from an acute increase in intracranial pressure. ${ }^{89}$ This may happen in fulminant cases of intracranial hypertension.

The positive Mantoux test and high ESR in our patient suggested tuberculosis infection; however, Quantiferon-TB gold in-tube test in our patient came back negative. A negative Quantiferon-TB gold in-tube test has to be interpreted with caution in TBM as $50 \%$ of culture confirmed TBM were found to have had a negative Quantiferon-TB gold in-tube test. ${ }^{4}$ The rapid diagnosis of TBM is crucial for a good clinical outcome. In our patient, a final diagnosis of TBM was finally made when the CSF PCR was positive for M. tuberculosis complex in which antituberculous treatment was commenced. The gold standard for diagnosis of TBM is with culture of M. tuberculosis in CSF. Culture results are slow as culture with Lowenstein-Jensen media takes about 8 weeks, while semiautomated radiometric culture systems such as Bactec 460 take about 6 weeks to culture M. tuberculosis. At present, the use of PCR for the detection of $M$. tuberculosis DNA in CSF is the widely used method as it is more rapid, sensitive and specific. It can detect fewer than 10 microorganisms and can be used to detect M. tuberculosis in clinical specimens. ${ }^{4}$ Recent meta-analysis found that the commercial nuclei acid amplification assays for the diagnosis of TBM had 98\% specificity and $56 \%$ sensitivity. ${ }^{4}$ There are other alternatives to the conventional PCR technique, and they include real-time PCR, isothermal, strain displacement or transcription-mediated amplification and ligase reaction. ${ }^{3}$ In our patient, the PCR technique used for detection of M. tuberculosis in the CSF was quantitative real-time PCR. The quantitative real-time PCR technique has been reported to have a higher sensitivity $(95.8 \%)$ and specificity $(100 \%)$ compared with the standard conventional PCR for patients with clinically suspected TBM. ${ }^{10}$

\section{Learning points}

- This case illustrates that ocular symptoms can be the only presenting feature of early stages of tuberculous meningitis (TBM) even though uncommon.

- Visual impairment, which usually occurs in paediatric population and late stages of adult TBM, occurred early in our patient.

- With that, we wish to highlight the atypical presentation of TBM and how with early recognition and treatment grave consequences of TBM can be averted.

Contributors $\mathrm{HZ}$ was involved in drafting the case report. SNL and SKV were involved in revising the case report. All three authors reviewed the case report before publication

Competing interests None declared.

Patient consent Obtained.

Provenance and peer review Not commissioned; externally peer reviewed (c) BMJ Publishing Group Ltd (unless otherwise stated in the text of the article) 2017. All rights reserved. No commercial use is permitted unless otherwise expressly granted.

\section{REFERENCES}

1 Lee HG, William T, Menon J, et al. Tuberculous meningitis is a major cause of mortality and morbidity in adults with central nervous system infections in Kota Kinabalu, Sabah, Malaysia: an observational study. BMC Infect Dis 2016;16:296.

2 Sinha MK, Garg RK, Anuradha Hk, et al. Vision impairment in tuberculous meningitis: predictors and prognosis. J Neuro/ Sci 2010;290(1-2):27-32.

3 Török ME. Tuberculous meningitis: advances in diagnosis and treatment. Br Med Bull 2015; 113:117-31

4 Thwaites G, Fisher M, Hemingway C, et al. British Infection Society guidelines for the diagnosis and treatment of tuberculosis of the central nervous system in adults and children. J Infect 2009;59:167-87.

5 Sher K, Firdaus, Abbasi A, et al. Stages of tuberculous meningitis: a clinicoradiologic analysis. J Coll Physicians Surg Pak 2013;23:405-812.

6 Marx GE, Chan ED. Tuberculous meningitis: diagnosis and treatment overview. Tuberc Res Treat 2011;2011:1-9.

7 Rowe FJ, Sarkies NJ. Assessment of visual function in idiopathic intracranial hypertension: a prospective study. Eye 1998;12:111-8.

8 Trobe JD. Papilledema: the vexing issues. J Neuroophthalmo/ 2011:31:175-86.

9 Abbasi HN, Brady AJ, Cooper SA. Fulminant idiopathic intracranial hypertension with malignant systemic hypertension-a case report. Neuroophthalmology 2013;37:120-3.

10 Takahashi T, Tamura M, Asami Y, et al. Novel wide-range quantitative nested real-time PCR assay for Mycobacterium tuberculosis DNA: clinical application for diagnosis of tuberculous meningitis. J Clin Microbiol 2008;46:1698-707.

Copyright 2017 BMJ Publishing Group. All rights reserved. For permission to reuse any of this content visit

http://group.bmj.com/group/rights-licensing/permissions.

BMJ Case Report Fellows may re-use this article for personal use and teaching without any further permission.

Become a Fellow of BMJ Case Reports today and you can:

- Submit as many cases as you like

- Enjoy fast sympathetic peer review and rapid publication of accepted articles

- Access all the published articles

- Re-use any of the published material for personal use and teaching without further permission

For information on Institutional Fellowships contact consortiasales@bmjgroup.com

Visit casereports.bmj.com for more articles like this and to become a Fellow 